\title{
Scalable interrogation: Eliciting human pheromone responses to deception in a security interview setting
}

\author{
Dr Alex W Stedmon ${ }^{\mathrm{a}^{*}}$, Dr Peter Eachus ${ }^{\mathrm{b}}$, Professor Les Baillie ${ }^{\mathrm{c}}$, \\ Dr Huw Tallis ${ }^{\mathrm{c}}$ Richard Donkor $^{\mathrm{d}}$, Robert Edlin-White ${ }^{\mathrm{d}}$ and Robert Bracewell ${ }^{\mathrm{d}}$ \\ ${ }^{a}$ Human Systems Integration Group, Coventry University, UK \\ ${ }^{\mathrm{b}}$ School of Social Work, Psychology and Public Health, University of Salford, UK \\ ${ }^{\mathrm{c}}$ School of Pharmacy and Pharmaceutical Sciences, University of Cardiff, UK \\ ${ }^{\mathrm{d}}$ Human Factors Research Group, University of Nottingham, UK
}

\section{${ }^{*}$ Corresponding Author: Dr Alex Stedmon}

Keywords: Hostile Reconnaissance, Terrorism, Deception, Pheromones, Stress, Security, Interrogation, Interviews

Highlights:

- for the first time a stress pheromone has been identified in a deception interrogation study

- this research validated a scalable interrogation paradigm

- raised levels of stress from participants in a deception condition were reflected across biological, physiological, psychological and behavioural measures

- measures provided robust data and an integrated understanding of differences in responses by those actively concealing information and those acting innocently

\begin{abstract}
Individuals trying to conceal knowledge from interrogators are likely to experience raised levels of stress that can manifest itself across biological, physiological, psychological and behavioural factors, providing an opportunity for detection. Using established research paradigms an innovative scalable interrogation was designed in which participants were given a 'token' that represented information they had to conceal from interviewers. A control group did not receive a token and therefore did not have to deceive the investigators. The aim of this investigation was to examine differences between deceivers and truth-tellers across the four factors by collecting data for cortisol levels, sweat samples, heart-rate, respiration, skin temperature, subjective stress ratings and video and audio recordings. The results provided an integrated understanding of responses to interrogation by those actively concealing information and those acting innocently. Of particular importance, the results also suggest, for the first time in an interrogation setting, that stressed individuals may secrete a volatile steroid based marker that could be used for stand-off detection. The findings are discussed in relation to developing a scalable interrogation protocol for future research in this area.
\end{abstract}

\section{Introduction}

Terrorist attacks are usually preceded by phases of 'hostile reconaissance' where those concealing their hostile intent enter public spaces in order to gather field intelligence (Linett, 2005). This intelligence is used to profile front-line security measures (e.g. staff rotas, security camera locations, emergency rendevous points) as well as wider systemic issues (e.g. 
response times to potential threats, communication between different security agents, and the wider organisation of security). From this intelligence it is then possible for terrorists to identify vulnerabilities in security and plan their attacks for maximum impact. Other criminal activities also adopt similar strategies to identify vulnerable people or locations (e.g. pickpockets targetting commuters at rush hour, graffiti artists exploiting security camera backspots). In all these situations if the would be criminal or terrorist are confronted, they will conceal their true intent in order not to raise any suspicion (Cain, 2009). However, it is during these instances that such cuplrits are at their most vulnerable, both to detection and disruption (Cain, 2009).

Given the risk of detection, individuals who are concealing any hostile intent may find the experience stressful particularly if they are intercepted and/or formally interviewed. Stress response data can be collected across behavioural, psychological, physiological and biological parameters (Selye, 1976). Whilst several studies claim to have identified the general presence of pheromones in participants who have been subjected to stressful experiences, the biology of stress in relation to chemo-signalling is less developed (Chen et al., 2006; Mujica-Parodi et al., 2008).

The role of pheromones in communication across the animal kingdom (e.g. insects, fish and mammals) is well documented (Chen and Haviland-Jones, 2000; Wyatt, 2003; Chen et al., 2006). Chemical communication by insects has shown that pheromones are used in attracting/detecting mates and when placed under stressful conditions, insects (e.g. bees and ants) are known to release alarm pheromones that warn others of impending dangers (Regnier and Law, 1968). Insect pheromones may be detected across large distances with moth pheromones being detected by other moths at distances in excess of five miles (Roach, 1975). Whilst it is apparent that animals use chemo-signalling, this form of vestigial olfactory communication for communicating stress, alarm or fear, is little known in humans (Ackerl et al., 2002). However, the effect of human scent on recipients is often subconscious (affective rather than cognitive) but still notable (Mujica-Parodi et al., 2009) and the human brain "automatically guides physiological adjustments to chemo-sensory anxiety signals without being dependent on conscious mediation" (Prehn-Kristensen et al., 2009, p.8). Furthermore, scent receiving participants cannot distinguish which nostril is receiving the scent illustrating that detection occurs within the olfactory system and not the trigeminal system (Zernecke et al., 2009).

Although there is considerable literature in deception and a little in human chemo-signalling, there is little or nothing on detecting deception through chemo-signalling (Eachus et al., 2013). The field is still in its infancy where much of the research is exploratory. A review by Mujica-Parodi et al., (2008) found only five previous studies investigating human alarm pheromones and none of these set out to create the specific and subtle cognitive state that might be experienced by someone attempting to conceal their intent when taking part in a suspect interrogation. Cognitive performance for a word association task illustrated that recipients of a 'fear' scent were more careful and more accurate than recipients of a neutral scent (Chen et al., 2006). Cognitive neuroscience experiments involving functional Magnetic Resonance Imaging (fMRI) scanning has illustrated activity changes in the amygdala, a structure activated in humans by emotional arousal and in animals by alarm pheromones (Mujica-Parodi et al., 2008). In other studies, recipients of a 'fear' scent were more likely to describe images of ambiguous facial expressions as 'fearful' (Zhou and Chen, 2009) and exhibited more extreme reactions to startle stimuli (Prehn et al., 2006). 
One of the inherent difficulties associated with the characterisation of human fear pheromones are their low concentrations in secreted media (Grosser et al., 2000). It is also assumed that alarm pheromones in humans must be volatile to ensure greater penetration of the olfactory membrane (Millar, 2002) and this may lead to practical difficulties in the capture, storage and analysis of samples. Expertise in methods and experimental protocols for this area is still developing. Some common themes in the design of the studies already cited include the use of the underarm axillary glands of males to collect scent samples (Zhou and Chen, 2009). Ferdenzi et al. (2009) found that differences between samples from left and right armpits and dependence on dominant-handedness were minimal. Donors are often expected to:

- abstain from the use of scented toiletries (Ackerl et al., 2002; Chen et al., 2006; Ferdenzi et al., 2009; Zhou and Chen, 2009)

- not use antiperspirant deodorants for two days before the study (Ackerl et al., 2002; Chen et al., 2006; Ferdenzi et al., 2009; Zhou and Chen, 2009; Prehn-Kristensen et al., 2009)

- be non-smokers or abstain from smoking during the study (Ackerl et al., 2002; Ferdenzi et al., 2009; Zhou and Chen, 2009; Prehn-Kristensen et al., 2009)

- abstain from drinking (Ackerl et al., 2002; Ferdenzi et al., 2009)

- avoid sport or sex (Ferdenzi et al., 2009)

- avoid odorous or spicy foodstuffs, cabbage or asparagus, garlic, onions before the study (Ackerl et al., 2002; Ferdenzi et al., 2009; Prehn-Kristensen et al., 2009)

- wash or shower with provided toiletries on the day of the study (Ackerl et al., 2002; Chen et al., 2006; Ferdenzi et al., 2009; Prehn-Kristensen et al., 2009)

- wear specific clothing (Ackerl et al., 2002; Zhou and Chen, 2009)

- complete questionnaires covering armpit shaving, oral contraception, menstrual cycle and medications (Ackerl et al., 2002)

- collect samples using self-administered oval cotton pads, taped on by the wearers for 24 hours (Ferdenzi et al., 2009)

- not be on medication or recreational drugs, have had no neurological, psychiatric, endocrine or immunological disease (Prehn-Kristensen et al., 2009)

- be of European origin (Prehn-Kristensen et al., 2009)

- be male donors (Zhou and Chen, 2009)

- use scent free toiletries and have washed their bed-sheets with odourless detergent (Zhou and Chen, 2009)

- have observed dietary restrictions and maintained dietary diary (Zhou and Chen, 2009)

Whilst these details are useful in establishing consistent protocols for new investigations, they present restrictions that can limit the ecological validity of such studies for many real life scenarios. It is also important to consider approaches to interviewing those suspected of deception. Guilty Knowledge Tests or Concealed Information Tests present multiple stimuli that include 'guilty' primes embedded within irrelevant knowledge. The responses to the guilty primes are then compared with the general baseline levels to identify differences in deception-based behaviours (Ganis and Patnaik, 2009). Interventionist interviewing approaches have also used, where unanticipated questions can help distinguish between liars and truth-tellers when there are two or more accounts of an event (Vrij et al., 2009). In another study, where participants were asked to recount an event in reverse chronological sequence, liars exhibited significantly more signs of cognitive load than the truth-tellers, and police officers were more able to distinguish liars from truth-tellers (Vrij et al., 2008). These 
perspectives provided the basis for investigating deception in scalable interviews where the level of questioning intensified. An integrated approach was adopted in order to understand and model deception across the four key factors. By taking this approach it was possible to investigate aspects of concealed intent from a user-centred perspective by incorporating data focused on user responses to the scalable interrogation process.

\section{Rationale}

A controlled laboratory study based on established research paradigms (Ackerl et al., 2002; Chen et al., 2006) utilised an innovative approach in which participants were required to actively deceive the investigator in return for a motivational reward. In order to achieve this some participants were given a 'token' that represented information they had to conceal when interviewed. A control group did not receive a token and therefore did not have to conceal information or deceive the investigators. The aim of this investigation was to determine if both sets of participants responded differently when interviewed, across the behavioural (e.g. video and audio recordings), psychological (e.g. subjective stress ratings), physiological (e.g. heart-rate, respiration, and skin temperature) and biological (e.g. cortisol levels and sweat samples) measures that were collected. As stress is a multi-factor concept, the measures were taken based on previous research protocols but also served as a basis for exploring the potential of a pheromone response to deception. This study was part of a larger investigation that also considered a field-based approach to modelling hostile intent and which discovered a stress related pheromone within a task that was analogous to conducting hostile reconnaissance (Eachus et al., 2013). The approach for the current study was analogous to a suspect who might be interviewed (by security representatives or the police) and who might actively withhold information or seek to deceive investigators in some way.

\section{Method}

\subsection{Participants}

In this study, an opportunistic sample of 38 male participants (mean age $=24$ years old) were recruited from staff $(n=6)$ and students $(n=32)$ at the University of Nottingham. Participants were predominantly right handed $(n=34)$. Male participants were recruited based on the view that males provide a better chemical response (Mujica-Parodi and Strey, 2006; Zhou and Chen, 2009). All participants were non-smokers, spoke English as their first language, were not taking any anti-depression/stress medication and had not been formally interviewed before by the Police.

\subsection{Apparatus}

Participants used an odourless soap to wash themselves in the $24 \mathrm{hrs}$ prior to the investigation. Baseline and interview questions were pre-recorded as digital (.wav) sound files that were then imported into a Microsoft Powerpoint presentation running on a standard laptop computer. The deception participants were issued with a specific 'token' that represented information they had to conceal. Due to the detailed nature of this study the specific measures are detailed below:

- Biological characteristics - levels of salivary cortisol were obtained using Sarstedt 'Salivette' testing kits, stored at $-20^{0} \mathrm{C}$ prior to analysis. In order to collect pheromone data, sweat samples were obtained from cotton pads secured under the armpits of participants with microporous tape. Following collection of the sweat samples, the pads from the participants dominant side were placed in $50 \mathrm{ml}$ sample 
tubes containing High Performance Liquid Chromatography (HPLC) grade Acetone $(10 \mathrm{ml})$ and stored at $-20^{\circ} \mathrm{C}$ for later analysis using Gas Chromatography-Mass Spectrometry (GC-MS).

- Physiological characteristics - physiological data were obtained using a wireless 'BioHarness' (Biopac Systems, Zephyr Technology Ltd) uploaded to a computer for analysis using AcqKnowledge software. Recordings were carried out in a constant temperature $\left(20^{\circ} \mathrm{c}\right)$ environment.

- Psychological factors - self-reported levels of stress were obtained using the short form of the State Trait Anxiety Inventory (STAI) questionnaire (Marteau and Becker, 1992). This is a six-item scale was derived from the original extended scale developed by Spielberger (1983). It required participants to record how they were feeling at that moment using a four point Likert scale.

- Behavioural factors - video and audio recordings of the baseline and interviews were collected for separate analysis as part of a complementary Engineering and Physical Research Council (EPSRC) project ('Shades of Grey': EP/H02302X/1).

\subsection{Design}

The study followed a $2 \times 3$ mixed design. The between-subjects independent variable had two levels: 'token' and 'no token' which corresponded to the concealment or non-concealment of information respectively. The within-subjects independent variable had three levels: 'baseline', 'interview 1' and 'interview 2' which corresponded to data collected at each stage of the scaled interrogation. The baseline session was designed to collect demographic and baseline data with the aid of relaxation based questions. The interviews were each designed to increase arousal from a low level (interview 1) to a higher level (interview 2) through the interrogation strategies employed. Interview 1 focused on issues of social desirability and withholding information whilst interview 2 focused more specifically on details of the token that a participant might have been concealing.

It was important that participants had specific information they had to conceal from the investigators. This was achieved by issuing the 'token' participants with information (Figure 1) that was designed to be easily remembered as a specific colour (yellow), a recognisable word (avocado) and a well-known landmark (the Eiffel Tower).

\section{Fig 1 about here}

The baseline and interview questions were pre-recorded so that they would be presented in a consistent manner, between-subjects. Participants were instructed that they would receive 20 questions from a larger sample. Whilst all interviews began with 'Question 1', they proceeded in a random order that made it difficult for participants to count the questions and anticipate the end of the interview. This, along with two interviewers conferring about the questions to use, gave the appearance that the questions were based on the specific responses that participants gave. What actually occurred was that all participants received the same 20 questions in each of the three conditions and the interviewers conferred in the same way on each occasion.

A number of dependent variable measures were taken across three of the four factors:

- Biological characteristics (cortisol data and stress pheromones) - these data were collected as a means of investigating biological traits of people concealing their intent. Cortisol is a validated measure of stress/arousal (Selye, 1974) and this was 
collected to support the biological validity of the study as well as provide support for the more innovative stress pheromone data.

- Physiological characteristics (heart-rate, respiration and skin temperature) - various data were collected in order to investigation the physiological profiles of participants who were concealing their intent. This approach was also used to help validate the design of increasing arousal levels through the scalable interrogation.

- Psychological responses (subjective measures of stress) - this approach and the measures used, was designed to assess the psychological state of participants across the different conditions of the scalable interrogation.

- Behavioural factors (video and audio data) - data from the baseline session and interviews were collected for separate analysis in the EPSRC 'Shades of Grey' project.

\subsection{Procedure}

The study progressed through three stages including: pre-trial preparation, baseline data collection, and the interview sessions. In the pre-trial preparation, participants were supplied with odourless medical soap to use on the day of the investigation, instructed to avoid using perfumes, body sprays and deodorants, and to refrain from alcohol, odorous food (such as curry), cabbage, and asparagus in the 24 hour period beforehand. During the study the thermal environment was controlled to maintain a consistent room temperature so as not to affect the physiological data.

During the baseline session the following activities were conducted where participants were required to:

- chew a salivette (to record cortisol levels), attach the under arm sweat pads and wireless BioHarness chest strap (to record sweat and physiological data).

- answer 20 pre-recorded baseline questions designed to relax them and also in order to collect basic demographic data

- complete the STAI and subjective ratings questionnaire

- remove sweat pads and chew another salivette

- the BioHarness data was downloaded and all other data labelled and stored.

During interviews 1 and 2 the following activities were conducted where participants were required to:

- attach fresh under arm sweat pads

- answer 20 pre-recorded questions

- remove sweat pads and chew another salivette

- the BioHarness data was downloaded and all other data labelled and stored

- complete the STAI and subjective ratings questionnaire.

After interview 2 participants were debriefed, signed a completion form and payment form. The experiment took approximately 75 minutes to complete. Participants were paid a combined inconvenience allowance and reward of $£ 30$ and provided with a deodorant stick after the study for personal refreshment. This payment was calculated to ensure good uptake of the study in the short timescales involved, to assure that the pre-trial preparations were followed correctly and also to act as an amount that would motivate participants to conceal information in interviews. 


\subsection{Ethics}

Before any data were collected the research proposal was considered by the University of Nottingham, Faculty of Engineering, Ethics Committee who were satisfied that the proposed research met the ethical standards required.

\section{4. $\quad$ Results}

The scalable interrogation study produced data across the four factors (e.g. biological, physiological, psychological and behavioural) however the behavioural data are not presented in this paper. Data were analysed using $2 \times 3$ mixed ANOVAs followed with post-hoc Bonferroni tests where required. Bonferroni tests were chosen as they are necessarily conservative in the face of multiple comparisons that might arise from mixed design analyses (Shaffer, 1995).

\subsection{Biological Characteristics}

Cortisol in saliva analysis: salivary cortisol samples were stored at $-20^{\circ} \mathrm{C}$ prior to analysis. The frozen salivettes were thoroughly thawed and centrifuged at $1000 \mathrm{x} \mathrm{G}$ for 2 minutes using an ELISA competitive kit (Salimetrics Ltd) with no modifications to the manufacturers guidelines. Four measures of salivary cortisol were obtained and analyses between each of them are represented as follows:

- Baseline - the difference between reading 1 and 2 for the start and end of the baseline session

- Interview 1 - the difference between reading 2 and 3 for the start and end of interview 1

- Interview 2 - the difference between reading 3 and 4 for the start and end of interview 2

The interval of time between obtaining the first and second samples of cortisol varied slightly within individual participants but was approximately 15 minutes and it was hypothesised that changes in cortisol levels during this period would be an indication of changes in arousal levels. The mean scores and standard deviations (Table I) illustrated that the relative changes in cortisol levels increased from baseline to interview 1 but and then deceased slightly in interview 2. A significant main was observed for 'session' $[F(2,72)=6.785, p<0.01]$ and post-hoc tests revealed a significant difference between baseline and interview 1 (mean difference $=-0.21, \mathrm{p}<0.01)$ illustrating that cortisol levels increased from baseline $(-0.01)$ to interview $1(0.20)$. No other significant effects were observed $(\mathrm{p}>0.05)$.

\section{Table I about here}

Further analysis revealed that 25 participants $(66 \%)$ demonstrated an increase in cortisol levels from interview 1 to interview 2; two participants $(6 \%)$ showed no change while the remaining 11 participants $(28 \%)$ presented a decrease in cortisol levels. Participants who were given the token were nearly twice as likely (15 of 18 participants; $83 \%$ ) to show an increase in cortisol levels compared to those no given a token (10 of 20 participants; 50\%). Of the remaining 10 individuals not given tokens two showed no change in cortisol whilst eight (40\%) presented decreased cortisol levels. All three of the individuals given tokens whose cortisol levels declined as a function of stress showed a substantial increase in the concentration of a potential stress pheromone in their sweat samples as the interrogations progressed. All three participants showed increases from the baseline profile to the stress profile of 5000 to 8000 units. This was significant as it showed that whilst cortisol levels 
may decrease in their saliva samples, when certain individuals are presented with stressful situations they have no control over the secretion of a stress pheromone in their sweat. Indeed the pheromone was released regardless of cortisol levels, when an individual is placed under stress. Therefore, the determination of whether a person is stressed or not may be more reliable by analysing their sweat rather than their saliva.

Stress pheromones: to determine the nature of the stress pheromone sweat samples collected in pads from the axilla of the dominant arm of participants during the Baseline, Interview 1 and Interview 2 were analysed using a Waters GCT Premier HP6890N GC machine. The analytes were desorbed in the injector port at a temperature of $200^{\circ} \mathrm{C}$. The mass spectrometer used was a Waters ESI with the detector in full scan mode (50-800 Da), relative to the lock mass (mass spectrometry grade heptacosa). The Mass spectrometer was equipped with a Nist MS Search 2.0 library searching facility.

Each profile showed the presence of several fatty acids, unsaturated hydrocarbons, as well as alcohols, esters and carboxylic acids. A peak was observed at a retention time of approximately 35.5 minutes for 37 participants (97.4\%) in the two interviews. An example of this relationship is represented in Figure 2 showing that from the baseline (green) responses to both interview 1 (blue) and interview 2 (red) were produced elevated levels of the pheromone at 35.5 minutes. These findings show that subjecting participants to scalable interrogations caused a noticeable increase in the magnitude of this peak, however, the peak is higher for interview 1 than interview 2 (showing a slight decline of approximately 15\%) in the relative intensity of this peak. The compound was only detected in trace amounts in all of the baseline samples analysed. Mass spectrum analysis of this peak identified the compound as Sitosterol. However, the presence of several fragments of higher mass in the spectrum suggests that Sitosterol is likely to be a breakdown product of a larger sterol that is fragmented, post collection, by the analysis process. There was some between-subjects variation in the levels of the pheromone and some participants showed a relative reduction in Sitosterol from interviews 1 to 2, suggesting that the overall response is non-linear.

\section{Fig 2 about here}

\subsection{Physiological Characteristics}

Heart rate: was sampled and edited at 50 to 240 beats per minute (BPM). Heart rate values were derived from the differences between R-R intervals. These were filtered from the ECG recorded data at a frequency of $1 \mathrm{~Hz}$ (1.008 Seconds). From a series of instantaneous heart rates recorded for $12 \mathrm{~min}$, mean and standard deviation heart rate were derived. Twenty seven samples (12 token holders and 15 no token participants) were analysed. The mean BPM values and standard deviation are illustrated in Table II. They illustrate a trend in the data that heart rate for token holders fell between the baseline and interview 1 but then rose during interview 2; whilst the opposite occurred for the no token holders as their heart rate increased during interview 1 but fell back to the baseline level in interview 2. Across the three session heart rate remained consistent but appeared higher for token holders when compared to no token participants. No significant effects were observed $(\mathrm{p}>0.05)$.

\section{Table II about here}

Respiration rate: data was measured in breaths per minute were acquired at a data frequency of $1 \mathrm{~Hz}$ (1.008 Seconds). Due to unusual peaks and troughs, respiration rate data from sensors 
responding to non-breathing related input (e.g. movement of the torso, speech, coughing) was filtered out. Minimum and maximum values were set to 0 and 70 respectively. Twenty seven samples (12 token holders and 15 no token participants) were analysed. The mean respiration rate values and standard deviations are illustrated in Table III. A significant interaction was observed for 'token $\mathrm{x}$ session' $[\mathrm{F}(2,50)=4.30, \mathrm{p}<0.05]$ illustrating that, respiration rates for token holders decreased as the investigation progressed. In contrast, higher respiration rates were recorded for no token participants.

\section{Table III about here}

The results for respiration were counter to expectations as faster breathing might indicate higher levels of arousal. Anecdotal evidence from the study suggests that some token holders made special efforts to control their breathing and heart rate, and that no token participants became annoyed at apparently being disbelieved and interrogated.

Skin Temperature: skin temperature was measured using the IR (infrared) sensor within the BioHarness device. Skin temperature data was acquired at $1 \mathrm{~Hz}(1.008$ Seconds) and measured between $10^{\circ} \mathrm{C}$ to $60^{\circ} \mathrm{C}$. Skin temperature data were analysed for all 38 participants (20 token holders and 18 no token participants). The mean skin temperature values and standard deviations are illustrated in Table IV. A significant main effect was observed for 'session' $[\mathrm{F}(2,72)=165.97, \mathrm{p}<0.001]$ and post-hoc tests revealed significant differences between baseline and interview 1 (mean difference $=1.366, \mathrm{p}<0.05$ ); baseline and interview 2 (mean difference $=1.837, \mathrm{p}<0.05$ ) and interview 1 and interview 2 (mean difference $=0.471$, $\mathrm{p}<0.05)$, illustrating that skin temperature increased significantly throughout the investigation.

\section{Table IV about here}

\subsection{Psychological Responses}

STAI questionnaires: were completed by participants at the start and end of each of the three conditions. Total scores ranged from 6 (minimum) to 24 (maximum). STAI data were analysed for all 38 participants (20 token holders and 18 no token participants). The mean and standard deviations are illustrated in Table $\mathrm{V}$ illustrating that self-reported levels of stress increased throughout the scalable interrogation and was also higher for the token holders.

\section{Table V about here}

A significant main effect was observed for 'session' $[\mathrm{F}(2,72)=6.619, \mathrm{p}<0.01]$ and post-hoc tests revealed significant differences between baseline and interview 2 (mean difference $=1.406, \mathrm{p}<0.05)$ illustrating that ratings were higher in Interview 2 (10.55) compared to Baseline (9.13). This finding suggested that participants in interview 2 experienced greater levels of stress, lending support to the validity of the scalable interrogation approach.

Subjective experiences: participants rated their experiences of interviews 1 and 2 based on six questions (Table VI). Responses were made on a 4-point Likert scale (where 1= 'strongly disagree' and $4=$ 'strongly agree') and were analysed using 2 (token) x 2 (session) mixed ANOVAs.

Table VI about here 


\section{Discussion}

By taking an integrated approach it has been possible to investigate laboratory based deception across a number of factors. Across all three factors analysed in this paper there were clear session effects that validate a laboratory based protocol for scalable interrogations.

Cortisol and sweat data were collected as an innovative means of investigating biological traits of people concealing their intent. Cortisol is a validated measure of stress/arousal and this was collected to support the biological validity of the pheromone data. The cortisol data sampled during the various investigation sessions revealed that in general terms, levels increased once an interrogation was underway. The sweat data also illustrated increased levels of Sitosterol (considered in more detail below).

Physiological data were collected in order to investigate any differences between the participant groups (e.g. deceivers and truth-tellers) and to support the validation of increasing arousal levels through interview 1 and interview 2 . Under increasingly difficult questioning, token holder respiration rates decreased whilst no token participant respiration rates increased. These results might indicate a degree of attempted behavioural control in the token holders under interrogation by the investigators. No other effects were observed between the token holders and no token participants but a number of effects were observed across the sessions (e.g. skin temperature increased throughout the study and heart rate remained constant).

In a similar way to the other measures, in order to investigate differences between the token holders and no token participants and any differences between the sessions based on increased arousal, it was important to assess the psychological state of participants via subjective measures. The responses illustrated that token holders rated the experience as increasingly stressful throughout the interrogation and in each session they rated the experience as more stressful than the no token holders). This supports the model of scalable interrogation but interview 2 was also rated as more stressful by the no token holders and this might also indicate that under increased questioning, the innocent no token participants may have felt that they were not being believed.

Although not a primary part of this paper, data was collected via video and audio recordings in order to investigate any observable physical actions, speech patterns and paralinguistic features in response to questioning. These data sets were transferred to the 'Shades of Grey' project for forensic analyses. Initial results of the audio data have indicated that the tokens holders increased their speaking rate whilst also significantly decreasing the response onset time and a reduction in hesitation phenomena suggesting an acceleration of overall speaking tempo (Kirchhubel, Stedmon and Howard, 2013).

That most of the statistically significant effects observed were related to session and fewer significant effects for token is not altogether surprising. The session variable was in a withinsubjects design, whereas the token variable was a between-subjects design and therefore inherently less statistically powerful, and more prone to compounding factors of human variability. The possibility of a within-subjects experimental design was explored but all approaches considered were rejected as being overly prone to order effects, especially learning effects. The selected design set out deliberately to create escalating levels of arousal/anxiety/concealment as the study progressed through the three sessions, and therefore the significant effects for session validate that aspect of the design. The presence or absence 
of a token was expected to have a subtle effect (compared to some of the previous 'fear' studies described earlier in this paper) and hence any significant effects are notable.

\subsection{Deception in experiments}

The more elements of deception in a study, the more scope there is for participants to have moments of realisation or changes in their levels of belief. As such, it may have been that some participants in the study did not believe their reward was at risk. However, this was controlled for in the question sequences for interviews 1 and 2. Participants were instructed that the questions would be selected specifically for them based on their responses. This process was designed to raise the level of stakes within the experiment so that participants did not feel the questions were predetermined regardless of their responses. In reality all participants received the same questions so that the questioning was standardized within the experiment.

Sip et al. (2008) identify common weaknesses in previous deception studies and make a series of methodological recommendations for future research. The most relevant to this study surrounds the construct of deception itself, and how or whether it can be adequately created in a laboratory study. Many studies involve 'instructions' to deceive or 'permission' to deceive and it can be argued that this creates different cognitive states and processes from genuine deception (by choice and without permission). It is generally very difficult to create precise cognitive states in a laboratory study and so a more sophisticated study might be based on a paradigm in which the participant is furtive by choice rather than deceptive by permission.

Hazlett (2005) reviewed research in detecting deception using behavioural indicators such as verbal, non-verbal and paralinguistic cues. He identified various limitations in current research:

- participants tend to be too calm and unmotivated, and have little personal stake in the outcome of deceit

- participants are often intelligent and well educated university students and staff from affluent backgrounds and often younger with limited life experience whereas criminals tend to be less intelligent and educated and well practiced in deception

The current study shares these characteristics to some extent. Indeed a specific selection criterion for participants was inexperience of police interviews, but this was chosen on ethical grounds and in order to investigate naïve participants rather than those with potentially criminal backgrounds and possible experience of real-life deception. In the future, and given the positive results across the factors outlined above, it would be valuable to extend the research into different areas of activity and across different population samples. A significant challenge is to create a study with a high sense of personal stake and motivation to deceive that still meets ethical protocols.

A further area of investigation would be the collection of similar data from female participants in order to identify if female deception can be detected in a similar manner to the male date that has been analysed. Linked to this, the wearing of body scents, deodorants and perfumes, as well as cultural differences and dietary factors could be investigated in order to assess the potential for collecting data from noisy samples or from those actively trying to disguise their pheromone response.

\subsection{A deception pheromone?}


Perhaps the most intriguing finding of this research is that Sitosterol, as one of several phytosterols, is present in human sweat and that has never been previously reported for a deception interrogation study. Its structure is very similar to that of cholesterol, which forms the basic structure of many other steroids found in the body. By comparing the mass spectrum of this larger unknown compound with the molecular ion values reported in the literature, we were unable to find a match. Further research is required to fully characterise this compound however it is fascinating to speculate that Sitosterol or its parent molecule may be a marker of a volatile steroid which could form the basis of a remote detection system for deception and stress.

Should a volatile alarm pheromone be characterised from human sweat it would pave the way for the development of detection devices to determine the presence and concentration of such a compound in an individual's odour. This technology would be invaluable in many areas including counter-terrorism and behavioural science as well as having a much broader appeal, as it would enable people to gauge the stress levels of an individual at a given time. Portable detection devices for volatile chemical compounds are already in use by forensic scientists (and military users to detect hostile gases), and most are based around GC-MS technology. Other approaches to field collection of volatile materials include Solid Phase MicroExtraction (SPME) which effectively traps volatile analytes in micro-fibres for analysis at a later time.

There are ongoing developments in alternative technologies for detecting subtle odour signals, using 'electronic noses' (Turner and Magan, 2004) or insects (Rains et al., 2008). Insect technologies (usually involving either contained or free-ranging honeybees, parasitic wasps or hawk moths) are sensitive; low cost compared to dogs or specialist laboratory equipment; quickly and easily trained ('conditioned') for particular smells; can be sacrificed or used in dangerous situations; and have potential uses in medicine, warfare, food safety, forensics (Rains et al, 2008). This is a very promising new 'technology' in its infancy. Notwithstanding this, most studies in the detection of cognitive states through odour have involved subjective human responses to odour samples. Regardless of the policies, procedures, or technologies that are developed to combat hostile intent, a methodology for testing the validity and reliability of different applications is required. The purpose of the research described here was to inform the development of a wider model of hostile intent (Eachus et al., 2013).

\section{Conclusion}

The research reported here has investigated the development of a model of deception in a laboratory setting and the relationship of a scalable interrogation protocol on concealed intent. The notion of deception was based on an abstract concept of withheld information (e.g. the token) that participants had to conceal from investigators. From the two interview sessions that were developed to probe and interrogate the participants, data across the four factor model of hostile intent provided robust measures and an integrated understanding of responses to interrogation by those actively concealing information and those acting innocently. These findings are all secondary to the primary aim of the research, which was to investigate a biological basis to deception. The finding that Sitosterol is present in human sweat as an otherwise unidentified compound associated with stress has been demonstrated for the first time in a deception interview setting. 


\section{Acknowledgement}

This research was funded by Home Office contract: CDE12123.

\section{References}

Ackerl, K., Atzmueller, M., and Grammer, K. (2002). The scent of fear. NeuroEndocrinology Letters 23, 79-84.

Cain, A. (2009). Can we hope to manage terrorism? Security Management Today (SMT) Online. http://www.ifsecglobal.com/can-we-hope-to-manage-terrorism/ (accessed: 03/06/14).

Chen, D., and Haviland-Jones, J. (2000). Human olfactory communication of emotion. Perceptual and Motor Skills, 91, 771-781.

Chen, D., Katdare, A., and Lucas, N. (2006). Chemosignals of fear enhance cognitive performance in humans. Chemical Senses, 31(5), 415-423.

Eachus, P., Stedmon, A.W., and Baillie, L. (2013). Hostile intent in public crowded spaces: A field study. Applied Ergonomics, 44(2013), 703-709.

Ferdenzi, C., Schaal, B., and Roberts, S.C. (2009). Human axillary odor: Are there siderelated perceptual differences? Chemical Senses, 34, 565-571.

Ganis, G., and Patnaik, P. (2009). Detecting concealed knowledge using a novel attentional blink paradigm. Applied Psychophysiology and Biofeedback, 34(3), 189-196.

Grosser, B.I., Monti-Bloch, L., Jennings-White, C., and Berliner, D.L. (2000). Behavioral and electrophysiological effects of androstadienone, a human pheromone.

Psychoneuroendocrinology, 25(3), 289-299.

Hazlett, G. (2005). Research on detection of deception: What we know vs. what we think we know. In, R. Swenson (Ed.) Educing Information/Interrogation: Science and Art/Foundations for the Future. Washington, DC, NDIC PRESS, 45-62.

Kirchhubel, C., Stedmon, A.W., and Howard, D.M. (2013). Analysing deceptive speech. In, D. Harris (Ed). Engineering Psychology and Cognitive Ergonomics: Understanding Human Cognition. Lecture Notes in Computer Science, 8019(2013), 134-141

Linett, H. (2005). Living with Terrorism: Survival Lessons from the Streets of Jerusalem, Boulder, Colarado: Paladin Press.

Marteau, T.M and Bekker, H. (1992). The development of a six-item short-form of the state scale of the Spielberger State-Trait Anxiety Inventory (STAI). British Journal of Clinical Psychology, 31, 301-306.

Millar, J.G. (2002). Sampling and sample preparation for pheromone analysis. In, J. Pawliszyn (Ed.) Sampling and Sample Preparation for Field and Laboratory. Amsterdam: Elsevier. 669-698. 
Mujica-Parodi, L., and Strey, H. (2006). Identification and Isolation of Human Alarm Pheromones. Report for U.S. Army Medical Research and Materiel Command, Fort Detrick, Maryland 21702-5012

Mujica-Parodi, L.R., Strey, H.H., Blaise F., Savoy, R., Cox, D., Ravindranath, B., and Botanov, Y. (2008). Second-hand stress: neurobiological evidence for a human alarm pheromone. Proceedings of the Second World Conference on Stress, 2007, Budapest.

Mujica-Parodi, L.R., Strey, H.H., Frederick, B., Savoy, R., Cox, D., Botanov, Y., Tolkunov, D., Rubin, D., and Weber, J. (2009). Chemosensory cues to conspecific emotional stress activate amygdala in humans. PLoS One, 4(7):e6415.

Prehn, A., Ohrt, A., Sojka, B., Ferstl, R., and Pause, B.M. (2006). Chemosensory anxiety signals augment the startle reflex in humans. Neuroscience Letters, 394, 127-130.

Prehn-Kristensen, A., Wiesner, C., Bergmann, T.O., Wolff, S., Jansen, O.,Mehdorn, H.M., Ferstl, R., and Pause, B.M. (2009). Induction of empathy by the smell of anxiety. PLoS One, 4(6): 55987.

Rains, G.C., Tomberlin, J.K., and Kulasiri, D. (2008). Using insect sniffing devices for detection. Trends in Bioltechnology, 26, 288-294.

Regnier, F.E., and Law, J.H. (1968). Insect pheromones. Journal of Lipid Research, 9, 541551.

Roach, S.H. (1975). Heliothis zea and H. virescens: Moth activity as measured by blacklight and pheromone traps. Journal of Economic Entomology, 68(1), 17-21.

Shaffer, J.P. (1995). Multiple hypothesis testing. Annual Review of Psychology, 46, 561-584.

Selye, H. (1976). Forty years of stress research: Principal remaining problems and misconceptions. Canadian Medical Association Journal, 115(1), 53-56.

Sip, K.E., Roepstorff, A., McGregor, W., and Frith, C.D. (2008). Detecting deception: The scope and limits, Trends in Cognitive Sciences 12(2), 48-53.

Spielberger, C.D. (1983). State-Trait Anxiety Inventory for Adults. Palo Alto, CA: Mind Garden.

Turner, A.P.F., and Magan, N. (2004). Electronic noses and disease diagnostics. Nature Reviews Microbiology. 2, 161-166.

Vrij, A., Mann, S., Fisher, R., Leal, S., Milne, B., and Bull, R. (2008). Increasing cognitive load to facilitate lie detection: The benefit of recalling an event in reverse order. Law and Human Behavior, 32, 253-265.

Vrij, A., Leal, S., Granhag, P.A., Mann, S., Fisher, R.P., and Hillman, J. (2009). Outsmarting the liars: The benefit of asking unanticipated questions. Law and Human Behavior, 33, 159166. 
Wyatt, T.D. (2003). Pheromones and Animal Behaviour: Communication by Smell and Taste. Cambridge: Cambridge University Press.

Zernecke, R., Kleemann, A.M., Haegler, K., Albrecht, J., Vollmer, B., Linn, J., Brückmann, H., and Wiesmann, M. (2009). Chemosensory properties of human sweat. Chemical Senses, $35(2), 101-108$.

Zhou, W., and Chen, D. (2009). Fear-related chemosignals modulate recognition of fear in ambiguous facial expressions. Psychological Science, 20, 177-183. 